\title{
On Equilibria in Duopolies with Finite Strategy Spaces
}

\author{
Kalyan Talluri *
}

July 18, 2003

\begin{abstract}
We will call a game a reachable (pure strategy) equilibria game if starting from any strategy by any player, by a sequence of best-response moves we are able to reach a (pure strategy) equilibrium. We give a characterization of all finite strategy space duopolies with reachable equilibria. We describe some applications of the sufficient conditions of the characterization.
\end{abstract}

*Kalyan Talluri, Department of Economics and Business, Universitat Pompeu Fabra, Ramon Trias Fargas 25-27, 08005 Barcelona, Spain, email: kalyan.talluri@upf.edu

Keywords: Duopoly, equilibria, revenue management, discrete-choice theory

JEL Classification: C0-Mathematical and Quantitative Methods: General;D4-Market Structure and Pricing;C7-Game Theory and Bargaining Theory 
Consider a game $G$ with 2 players with finite strategic spaces $1, \ldots, n_{1}$ and $1, \ldots, n_{2}$ respectively. We define a directed bipartite graph, called the $b-r$ graph, representing the game as follows: Let $G=\left(N_{1}, N_{2}\right)$ be a directed bipartite graph with $n_{1}$ nodes in $N_{1}$ and $n_{2}$ nodes in $N_{2}$ and an $\operatorname{arc}(i, j)$ if $j$ is a best response to player 1's strategy $i$. We will call the arcs of $G$ best response arcs or $b$-r arcs. Note that every node can have multiple outgoing b-r arcs, and has at least one such outgoing b-r arc (as we assume each player has at least one best response to every strategy in the other player's strategy space).

An equilibrium is a pair of arcs of the form $(i, j)$ and $(j, i)$. We will say $G$ is a graph with reachable equilibria if every node in $G$ has a directed path to an equilibrium pair of arcs. In the game, this would correspond to being able to reach an equilibrium by a sequence of best responses starting from any strategy.

\section{Main Result}

Note that the nodes of $N_{1}$ and $N_{2}$ can be labeled $1, \ldots, n_{1}$ and $1, \ldots, n_{2}$ any way we want. It does not affect whether $G$ has an equilibrium or not in any way. Any such labeling forms a linear order on the sets $N_{1}$ and $N_{2}$ respectively. For a given such labeling we will say two b-r arcs $\left(i_{1}, i_{2}\right)$ and $\left(j_{1}, j_{2}\right)$ are said to cross if $i_{1}<j_{1}$ and $j_{2}<i_{2}$ in the linear order defined by the labeling. Given a labeling of $N_{1}$ and $N_{2}$ we define a subgraph $H$ of $G$, called the lowest b-r arcs graph, given by each player's lowest best response strategy in the linear ordering of the labeling to each strategy of the other player. For example, if in a particular labeling of the nodes, player 2's best response to player 1's move of $i$ is $j_{k_{1}}<\cdots<j_{k_{m}}$, then $H$ will contain just the one b-r arc $\left(i, j_{k_{1}}\right)$. Note that $H$ represents a particular choice of best response functions from the best response correspondences represented by $G$.

Our main result is the following:

Theorem $1 G$ is a reachable equilibria game if and only if there exists a labeling (i.e., linear ordering) of $N_{1}$ and $N_{2}$ of the b-r graph $G$ such that the arcs in $H$, the lowest b-r graph, are non-crossing.

Before we give a proof of the theorem, we will make a few comments on its implications:

- Theorem 1's interpretation should be that in order to prove existence of equilibria in a duopoly with finite strategy spaces, in most cases, it would be sufficient to just try and find an appropriate linear order and prove that there are no crossing arcs. Theorem 1 also tells us that we can limit ourselves to considering only the lowest b-r arcs.

- While Theorem 1 applies only to duopolies, it could also be viewed, in the negative sense, as a result on oligopolies: i.e., if one cannot find such linear orderings of the strategy spaces for the 2-payer version of a given oligopoly game, it is unlikely we will be able to prove the existence of a reachable equilibria for the given oligopoly game. 


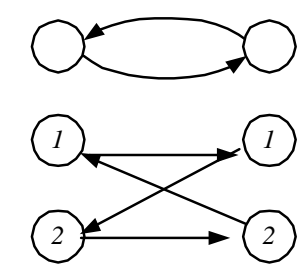

Figure 1: A graph with an equilibrium but no non-crossing ordering of nodes.

- For equilibria that are not reachable, it is unlikely that one would be able to say something in the style of Theorem 1. Consider the game given by the graph in Figure 1. The equilibrium is not reachable and there is no non-crossing labeling possible.

- For continuous but bounded strategy spaces, a sufficient discretization can make the theorem applicable.

$\underline{\text { Proof }}$

(if): The sufficiency part is very easy to prove (indeed, existence follows directly from Tarski's fixed point theorem applied to linear ordered sets; but the following elementary graph-theoretical argument illustrates the concept of reachable equilibria): Consider the non-crossing labeling and starting from any node of $N_{1}$, follow a sequence of best-responses given by the subgraph $H$. Since the number of nodes is finite, either we end up at an equilibrium pair of arcs, or we end up doubling back and creating a crossing pair of arcs, a contradiction.

(only if): The necessity is the surprising part, and also the more difficult.

Partition the set of nodes of $G$ by the following process. Start off with an equilibrium pair $(i, j)$ (call it root equilibrium pair) and consider the set of nodes $S_{1}$ consisting of all nodes from which we can reach $i$ or $j$ (basically set of all nodes with directed paths in $G$ to $i$ or $j$ ). Remove all the nodes of $S_{1}$ and repeat (say $k$ times) till there are no more nodes left. Since $G$ is a reachable equilibria graph, it should be clear that $S_{1} \cup \cdots \cup S_{k}$ forms a partition of $N_{1}, N_{2}$. Moreover, note that there is no b-r arc in $G$ that goes from a node in $S_{j}$ to a node in $S_{i}$ if $i<j$.

Now compress the nodes in $S_{1}, \ldots, S_{k}$ to form a new graph $K$ with nodes $\{1, \ldots, k\}$ corresponding to $S_{1}, \ldots, S_{k}$. Notice that there cannot be cycles in this graph $K$ by the following argument: Suppose there was a cycle. Pick the lowest indexed node in the cycle. It has an incoming arc from a node with a higher number which would contradict the fact that there is no b-r arc in $G$ that goes from a node in $S_{j}$ to a node in $S_{i}$ if $i<j$. So $K$ is an acyclic graph, and by a basic result in graph theory, the nodes of $K$ have a labeling such that for every arc $(i, j), i<j$. We will just assume without loss of generality that $S_{i}, i=1, \ldots, k$ are ordered as such. Call this the ordering of the sets of the partition.

Now consider the partition $S_{i}, i=1, \ldots, k$. We will show that within each of these 
sets of the partition, we can order the nodes appropriately (ordering within the sets of the partition). This combined with the above ordering of the sets of the partition will give us the appropriate linear ordering that we seek.

Consider a set $S \in\left\{S_{1}, \ldots, S_{k}\right\}$. Order the elements of $S$ by the following algorithm: Let $T^{(0)}$ be the subgraph consisting of the root equilibrium pair of $S,(i, j)$. These two nodes will be the lowest ranked nodes in $S \cap N_{1}$ and $S \cap N_{2}$ respectively.

Consider the steps in the following algorithm. At stage $m, T^{(m)}$ will be a subgraph with a linear order of nodes in $T^{(m)} \cap N_{1}$ and $T^{(m)} \cap N_{2}$. Moreover, the set $T^{(m)}$ will be "closed" in the sense that it will be a reachable equilibria graph, and the lowest arc subgraph of $T^{(m)}$ has no crossing arcs (indeed, $T^{(m)}$ will have exactly one out going arc per node, so when we say lowest arc subgraph, it is with respect to $S$ ). Trivially, this is true for $T^{(0)}$.

Given $T^{(m)}$, we construct $T^{(m+1)}$ by the following process: Let $\left\{1, \ldots, p_{m}\right\}$ be the nodes in $T^{(m)} \cap N_{1}$. For $i=1, \ldots, p_{m}$, pick all $j \notin T^{(m+1)}$ and arc $(j, i) \in S$ and add them to the set $T^{(m+1)}$. Moreover give them a linear order according to the order in which they were added to $T^{(m+1)}$. Similarly, for $\left\{1, \ldots, q_{m}\right\}$, the nodes in $T^{(m)} \cap N_{2}$. That brings us the end of stage $m+1$. Repeat till $m=M$ where all the nodes of set $S$ are included in $T^{(M)}$.

Clearly $T^{(m+1)}$ is a reachable equilibria subgraph if $T^{(m)}$ is, as we can reach the root equilibrium pair of $S$ from the newly added nodes. It only remains to show that we do not create any crossing lowest b-r arcs going from stage $m$ to $m+1$. Suppose we have added arc $\left(i_{1}, i_{2}\right)$ in building $T^{(m+1)}$, and say it crosses with $\left(j_{1}, j_{2}\right) \in T^{(m+1)}$. First, $\left(i_{1}, i_{2}\right)$ is a lowest b-r arc as if $i_{1}$ had an arc to a node in $1, \ldots, i_{2}-1$, it would have been added sooner. Next, if it crossed $\left(j_{1}, j_{2}\right) \in T^{(m+1)}$, it means that the current linear order (the order of the nodes of $T^{(m+1)}$ just prior to adding $\left.\left(i_{1}, i_{2}\right)\right)$ has $i_{1}>j_{1}$, as $\left(j_{1}, j_{2}\right)$ existed in $T^{(m+1)}$ before $\left(i_{1}, i_{2}\right)$; and as it is crossing, $i_{2}<j_{2}$. But if $i_{2}<j_{2},\left(i_{1}, i_{2}\right)$ should have been added to $T^{(m+1)}$ earlier than $\left(j_{1}, j_{2}\right)$, a contradiction. Figure 2 shows this construction process.

Repeating this for each set $S \in\left\{S_{1}, \ldots, S_{k}\right\}$, we have an ordering for each $\left\{S_{1}, \ldots, S_{k}\right\}$ and subgraphs $\left\{T_{1} \subseteq S_{1}, \ldots, T_{k} \subseteq S_{k}\right\}$ that represent the subgraph of lowest non-crossing b-r arcs within the subsets of the partition.

Finally, there remains the task of stitching together the orders within the sets of the partition with the orders of the sets of the partition. For this, just consider the lexicographic order on node $i$ as $\left[s_{i}, t_{i}\right]$, where $s_{i}$ is the rank of the set in the partition containing node $i$, and the $t_{i}$ is the rank of the node within the set of the partition. This lexicographic ordering creates a linear ordering of the nodes of $N_{1}$ and $N_{2}$. We have to show that the statement of the theorem holds for this lexicographic ordering. We will do this by showing that $H$, the lowest b-r subgraph of $G$ in this lexicographic ordering is exactly $T_{1} \cup \cdots \cup T_{k}$.

Arcs of the form $(i, j) \in S_{l} \cap G$ and $(i, j) \notin T_{l}$, clearly are not lowest b-r arcs. Suppose an $\operatorname{arc}(i, j)$ is such that $i \in S_{n}$ and $j \in S_{l}$. Let $(i, t)$ be the lowest b-r arc in $T_{n}$.

Then in the compressed graph $K$, there would have been an arc $(n, l)$, implying $n<l$ in the order of the partitions. Hence, $(i, j)$ cannot be a lowest b-r arc because in the lexi- 


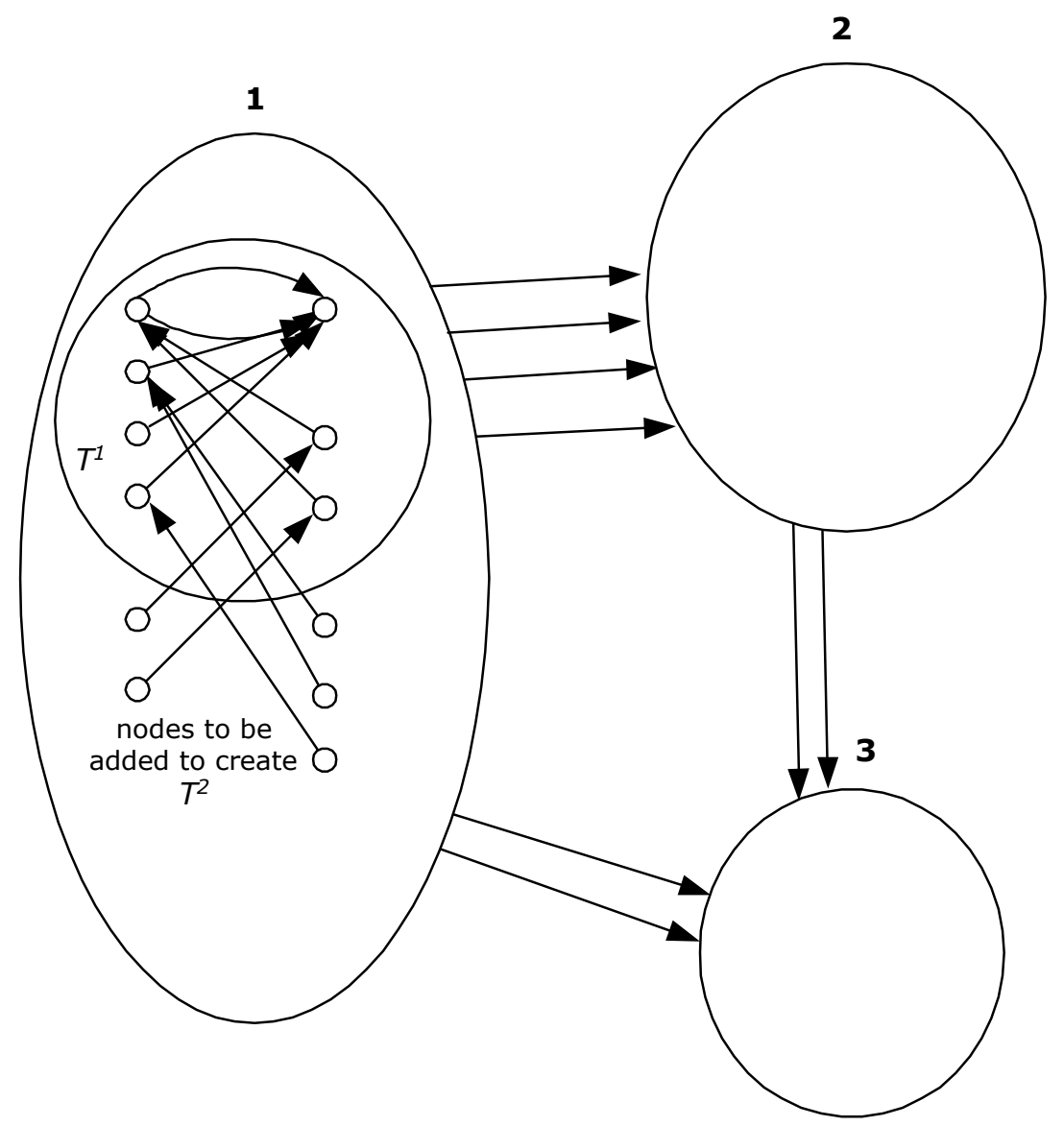

Figure 2: Labeling the nodes starting from an equilibrium pair of arcs. The labeling of the partition is shown as bold numbers. Within a set of the partition, a "braid" like structure of arcs results from the labeling process.

cographic ordering, node $t$ would have a lower rank than node $j$.

Theorem 1 does not provide any hint about uniqueness. Indeed, giving useful, nontrivial, characterizations of uniqueness seems more difficult than of existence. We do not have even strong sufficient conditions, although given the simple nature of the graphs, we are optimistic in this regard.

We give below some rather weak and obvious conditions.

Corollary 1 The game $G$ has an unique equilibrium if there is a unique best-response for each strategy of the other player, $G$ is connected, and there is a linear order such that the b-r graph $H$ has non-crossing arcs.

In the above, unique equilibrium means the game has a single pure-strategy equilibrium. 
The game can have multiple equilibria but a unique reachable equilibrium, which we define as an equilibrium that is reachable from any strategy by any player by a sequence of bestresponse moves.

The following proposition will also be used in our later application:

Proposition 1 Let the set of nodes $S_{1}$ of $G$ be such that no node in $S_{1}$ is a best response arc to any strategy of firm 2, and similarly $S_{2}$ for firm 2. Suppose $G^{\prime}=N_{1}-S_{1}, N_{2}-S_{2}$ have a labeling such that the arcs of the lowest b-r graph of this labeling, H', are non-crossing. Then there exists a labeling of $G$ such that its lowest b-r graph $H$ has non-crossing arcs. a labeling of $G$

$\underline{\text { Proof }}$

Consider a node $i$ in $S_{1}$. Let $j$ be the best-response node in $N_{2}-S_{2}$ corresponding to $i$. Let $b^{-1}(j)$ be the node of firm 1 such that the best response arc in $H \prime$ of firm 1 's $b^{-1}(j)$ strategy has the largest label less than or equal to the label of $j$. Give node $i$ the label of $b^{-1}(j)$ plus one, and add one to all the labels of nodes with labels greater than that of $b^{-1}(j)$. Repeat this procedure for all nodes in $S_{1}$ and $S_{2}$. The relative position of the nodes of $N_{1}-S_{1}$ and $N_{2}-S_{2}$ are not changed and the new arcs do not cross with any existing lowest b-r arcs.

\subsection{Supermodularity}

Equilibria in supermodular games is a well-studied topic with a wide variety of applications. This says that if the payoff functions for both players (note, the statement is with respect to the pay off functions and not the best response functions) are supermodular and satisfies some properties, then the game has an equilibrium.

Here we repeat the definitions from Vives [10] (see [10] for definitions of lattice etc.). A game is supermodular if for each player $i$, the strategy space $A_{i}$ is a complete lattice, the payoff function $\pi_{i}$ is upper semicontinuous and supermodular in player $i$ 's strategies $a_{i}$, i.e.,

$$
\pi_{i}(\inf (x, y))+\pi_{i}(\sup (x, y)) \geq \pi_{i}(x)+\pi_{i}(y), \forall x, y \in A_{i}
$$

for a fixed set of strategies of all other players $a_{-i}$, and displays an increasing differences property in $\left(a_{i}, a_{-i}\right)$. The increasing differences property is as follows (again from Vives [10], but specialized to linearly ordered sets): Let $N_{1}$ and $N_{2}$ be two finite sets. The function $\pi: N_{1} \times N_{2} \rightarrow \Re$ has increasing differences in its two arguments $(i, j)$ if $\pi\left(i, j_{1}\right)-\pi\left(i, j_{2}\right)$ is increasing in $i$ for all $j_{1} \geq j_{2}$. Topkis [9] showed that all supermodular games have an equilibrium. For the linearly ordered case of course, Equation 1 is redundantly true for all pay-off functions, so we need to consider only the increasing differences property.

For our discrete, finite strategy space case, supermodularity and the the increasing differences property implies non-crossing arcs, but our results do not require supermodularity. Essentially the increasing differences property says (in our graph-theoretical terminology) 

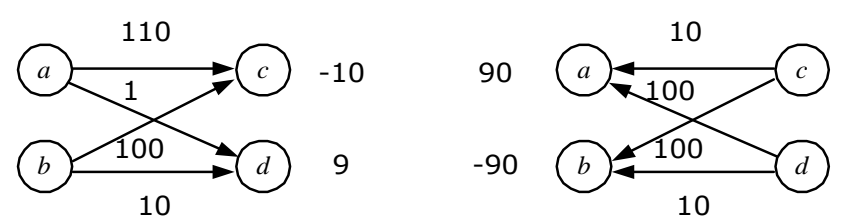

Figure 3: The graph on the left represents the payoffs for player 2 and the one on the right, those for player 1. Game has an equilibrium, but payoff functions (under any ordering) do not have the increasing differences property (the numbers next to the nodes represent $\pi_{2}(\cdot, b)-\pi_{2}(\cdot, a)$ and $\left.\pi_{1}(\cdot, d)-\pi_{2}(\cdot, c)\right)$.

that for a fixed ordering if $\left(i_{1}, i_{2}\right)$ and $\left(j_{1}, j_{2}\right)$ are crossing arcs, then 1 is better off using $\left(i_{1}, j_{2}\right)$ and $\left(j_{1}, i_{2}\right)$ instead, so no best-response arcs cross. This is then shown to be sufficient for the game to have a pure strategy equilibrium.

Our result is slightly more general (albeit only for finite, linearly ordered structures) in the sense, we do not specify why the game should not have crossing b-r arcs.

Figure 3 shows an example where the payoff functions do not have the increasing differences property (under any linear ordering), yet there is an equilibrium. Our sufficiency condition however, would imply the existence of an equilibrium, because we consider only non-crossing of best-response functions. Whether something like this example will come to pass in a real "interesting" application, we do not know yet, and till we encounter some actual application, we will hold off judgment on the degree of generalization this new framework provides. In any case, we see the converse direction as the more surprising and interesting result of this research.

\section{Applications}

Although Theorem 1 does not specify a priori what the linear ordering of the strategies should be, in many applications the natural ordering suggested by the problem works. Indeed, this is the case for the applications in Lippman and McCardle [5], Netessine and Shumsky [6] (see Talluri and van Ryzin [8]) and Cachon [3].

Discrete and finite strategy spaces also come up naturally in applications (and not just as approximations to continuous models. Indeed the problems in [5], [6] and [3] are more naturally stated in terms of discrete strategies in units of inventory).

We give below an application from revenue management where a continuous approximation is not possible, but equilibria can be proved very easily using our graph-theoretic techniques.

Assume that two airlines sell their inventory for their flights (one flight each, called resource) at multiple prices (a product is a reservation for future usage of one unit of the 
airline capacity; in addition multiple products are created by sale restrictions - such as cancellation and advance purchase restrictions. Customers self-select their products.).

Firms fix the prices for the duration of the sale of their inventories, and only change allocations to the different products. This is how quantity based revenue management is practiced in the airline industry (besides hotels, railway and other industries). Such static, fixed, prices are preferred when prices have to be advertised, or resources are sold based on reservations, or it is otherwise costly to change prices.

In our model time is discrete and there are $T$ units of time till the usage of the resource. The resources of both firms are consumed simultaneously at time $T$. Bookings happen during the intervals 0 to $T$ with at most one arrival during each period. A consumer arrives in a period, observes the available choices of the two firms and then, based on the prices and attributes of the fare products, either decides to buy one of the products of one of the firms, or decide not to purchase any of the available products (let 0 represent this no-purchase alternative). We assume a multinomial logit model (see Ben-Akiva and Lerman [2]), with a no-purchase alternative, as the consumer's choice rule. In each period there is a probability $\lambda$ of a consumer arrival. If a consumer does not purchase in a period, he disappears; i.e., he does not reappear in a later period. So, the consumer is passive and does not play any strategic game to maximize his utility.

Since the choice rule is multinomial logit, a customer's probability of choosing an available product $j$ of firm $i$, when firm $i$ offers the set $A^{i}$ can be represented for convenience by:

$$
\gamma_{j}^{i}\left(A^{1}, A^{2}\right)=\frac{w_{j}^{i}}{\sum_{j \in A^{1}} w_{j}^{1}+\sum_{j \in A^{2}} w_{j}^{2}+w_{0}},
$$

where the weights $w_{j}^{1}$ and $w_{j}^{2}$ could possibly be functions of the prices $p_{j}^{1}$ and $p_{j}^{2}$ and other attributes, and $w_{0}$ is the weight of the no-purchase alternative.

Firms in turn start off with capacities of $C_{1}$ and $C_{2}$ units of inventory and $n_{1}$ and $n_{2}$ set of products respectively. They fix the prices of the products $p_{1}^{1}, p_{2}^{1}, \ldots, p_{n_{1}}^{1}$ and $p_{1}^{2}, p_{2}^{2}, \ldots, p_{n_{2}}^{2}$ and keep them fixed throughout the booking period. At the beginning of each period, each firm makes available a subset of its fare products. So note that even though prices are fixed, the firms can effectively change the prices by deciding on what subset they make available simultaneously. All in all, while one can criticize the specificity of the logit functional form, this model captures both customer choice and the dynamics of a revenue management problem quite accurately. There are two informational assumptions one can make: firms do not observe each other's remaining capacity; firms observe remaining capacity. In either case, if a firm sells out all capacity, then it can only offer a null set, and its competitor realizes that it has sold out. 


\subsection{Observable capacities}

In this case, the state space includes competitor's capacities. So the reaction function is based on one's own remaining capacity, competitor's remaining capacity, and competitor's current offer set.

It is shown in Talluri and van Ryzin [7] that the multinomial logit customer choice model, for the monopoly case, has the nested-by-fares property which means that each firm needs to consider only complete sets, that is, for firm 1 , sets of the form $\left\{1,2, \ldots, k_{1}\right\}$, for $k_{1}=1,2, \ldots, n_{1}$ and for firm 2 , sets of the form $\left\{1,2, \ldots, k_{2}\right\}$, for $k_{2}=1,2, \ldots, n_{2}$. We will assume here that the strategy spaces are indeed these complete sets. In practice, most airlines use nested controls and nested offer sets. We will represent such complete sets compactly as $A_{k_{1}}$ and $A_{k_{2}}$ respectively, and the collection of all such sets $\mathcal{N}_{1}$ for firm 1 and $\mathcal{N}_{2}$ for firm 2. We will say, set $A_{k_{1}}<A_{k_{2}}$ if $k_{1}<k_{2}$ or as the sets are nested, $A_{k_{1}} \subset A_{k_{2}}$. If $A_{k_{1}}<A_{k_{2}}$, we will denote the set difference as $A_{k_{2}}-A_{k_{1}}$.

The value function for firm 1 at time $t$ given firm 2 offers $A^{2}$ is given by the following:

$$
\begin{aligned}
& V_{t}^{1}\left(x_{1}, x_{2} \mid A^{2}\right)= \\
& \quad \max _{A^{1} \subseteq \mathcal{N}_{1}}\left\{\lambda \sum_{j \in A^{1}} \gamma_{j}^{1}\left(A^{1}, A^{2}\right) p_{j}^{1}\right. \\
& +\left(1-\lambda+\lambda \gamma_{0}\left(A^{1}, A^{2}\right)\right) V_{t+1}^{1}\left(x_{1}, x_{2}\right) \\
& +\lambda \sum_{j \in A^{1}} \gamma_{j}^{1}\left(A^{1}, A^{2}\right) V_{t+1}^{1}\left(x_{1}-1, x_{2}\right) \\
& \left.+\lambda \sum_{j \in A^{2}} \gamma_{j}^{2}\left(A^{1}, A^{2}\right) V_{t+1}^{1}\left(x_{1}, x_{2}-1\right)\right\}
\end{aligned}
$$

where, $V_{t+1}(\cdot, \cdot)$ is the equilibrium revenue-to-go from period $t+1$ onwards.

There are two things to note about (2): (i) It is defined at time $t$ only if there is an equilibrium from $t$ till $T$. (ii) If there are multiple equilibria, we exogenously assume that firm 1 starts off with a specific strategy (say the strategy labeled 1 and by a sequence of best responses, both firms will end up at an equilibrium (i.e., the value function is again uniquely specified).

The value function for firm 2 is defined similarly. We will let $V_{t}^{1}\left(x_{1}, x_{2} \mid A^{1}, A^{2}\right)$ denote firm 1's revenue if it uses $A^{1}$ to react to firm 2's $A^{2}$.

While Equation 2 looks complicated, in words, it just says, given firm 2 chooses $A^{2}$ to offer, firm 1's revenue-to-go function is current period's revenue plus the revenue depending on what state they end up at the end of the period (by no sale, sale for firm 1, or sale for firm 2).

First of all, let us rewrite Equation 2, using the fact that

$$
\sum_{j \in A^{2}} \gamma_{j}^{2}\left(A^{1}, A^{2}\right)=1-\gamma_{0}\left(A^{1}, A^{2}\right)-\sum_{j \in A^{1}} \gamma_{j}^{1}\left(A^{1}, A^{2}\right),
$$


as:

$$
\begin{aligned}
& V_{t}^{1}\left(x_{1}, x_{2} \mid A^{2}\right)= \\
& \quad \max _{A^{1} \subseteq \mathcal{N}_{1}}\left\{\lambda \sum_{j \in A^{1}} \gamma_{j}^{1}\left(A^{1}, A^{2}\right)\left[p_{j}^{1}+V_{t+1}^{1}\left(x_{1}-1, x_{2}\right)-V_{t+1}^{1}\left(x_{1}, x_{2}-1\right)\right]\right. \\
& \quad+(1-\lambda) V_{t+1}^{1}\left(x_{1}, x_{2}\right) \\
& \left.\quad+\lambda \gamma_{0}\left(A^{1}, A^{2}\right)\left[V_{t+1}^{1}\left(x_{1}, x_{2}\right)-V_{t+1}^{1}\left(x_{1}, x_{2}-1\right)\right]\right\} \\
& \quad+\lambda V_{t+1}^{1}\left(x_{1}, x_{2}-1\right) .
\end{aligned}
$$

Let $\Delta=V_{t+1}^{1}\left(x_{1}, x_{2}-1\right)-V_{t+1}^{1}\left(x_{1}-1, x_{2}\right)$ and $\delta=V_{t+1}^{1}\left(x_{1}, x_{2}-1\right)-V_{t+1}^{1}\left(x_{1}, x_{2}\right)$. Let $g\left(\cdot \mid x_{1}, x_{2}\right)$ represent the term

$$
g\left(A^{1} \mid x_{1}, x_{2}\right)=\sum_{j \in A^{1}} w_{j}^{1}\left[p_{j}^{1}-\Delta\right]-w_{0} \delta .
$$

For simplicity we will just write $g\left(A^{1} \mid x_{1}, x_{2}\right)$ as $g\left(A^{1}\right)$, whenever there is no room for confusion. Notice that $g(\cdot)$ for firm 1 is independent of the strategies of firm 2 , and vice versa for firm 2 .

The following are intuitive and not hard to show rigorously:

$$
\begin{aligned}
& V_{t+1}^{1}\left(x_{1}, x_{2}\right) \leq V_{t+1}^{1}\left(x_{1}, x_{2}-1\right), \\
& V_{t+1}^{1}\left(x_{1}-1, x_{2}\right) \leq V_{t+1}^{1}\left(x_{1}, x_{2}\right),
\end{aligned}
$$

and

$$
V_{t+1}^{1}\left(x_{1}-1, x_{2}\right) \leq V_{t+1}^{1}\left(x_{1}, x_{2}-1\right) .
$$

Note that $\Delta-\delta=V_{t+1}^{1}\left(x_{1}, x_{2}\right)-V_{t+1}^{1}\left(x_{1}-1, x_{2}\right) \geq 0$.

As prices $p_{j}^{i}$ are decreasing in $j$, as firm i offers larger sets, the function $g\left(A^{i}\right)$ increases monotonically first and then decreases monotonically (that is, it is unimodal). The function $g\left(A^{i}\right)$ can also be negative. It could be the case that $g(\cdot)$ is negative for all strategies of firm $i$. Figure 4 shows the two possibilities for firm $i$, where $\bar{G}^{i}$ is the set that has the maximum value of $g$, and if the firm. We will call the case where $g\left(\bar{G}^{i}\right) \geq 0$ as Case I, and Case II when $g\left(\bar{G}^{i}\right)<0$.

Form an equilibrium bipartite graph with $n_{1}$ nodes on one side and $n_{2}$ nodes on the other side, with each node corresponding to each of the complete sets (Figure 7). The order of the nodes will be specified later depending on whether the firms are in Case I or Case II. For a given ordering any time $t$, firm 1 offers the set $k_{1}$, firm 2 has one (or more) best-response sets $k_{2}$. Represent each such best-response as an arc that originates at node $k_{1}$ and terminates at the node that represents firm 2's best response set. Similarly for all sets of firm 1 and vice versa for the best responses of firm 1 to the offer sets of firm 2 . So we have a directed bipartite graph where each node has at least one outgoing arc (and possibly zero, one or multiple incoming arcs). 
Lemma 1 If a firm, say firm 1 , is in Case I, a set $A^{1}$ with $g\left(A^{1}\right)$ negative, or $g\left(A^{1}\right)<$ $g\left(\bar{G}^{1}\right), A^{1}>\bar{G}^{1}$, is never the best responses to any strategy choice of the competitor.

$\underline{\text { Proof }}$

Let $A^{1}$ be such that $g\left(A^{1}\right)$ is negative. Then,

$$
\frac{g\left(A^{1}\right)}{\sum_{j \in A^{1}} w_{j}^{1}+\sum_{j \in A^{2}} w_{j}^{2}+w_{0}} \leq \frac{g\left(\bar{G}^{1}\right)}{\sum_{j \in \bar{G}^{1}} w_{j}^{1}+\sum_{j \in A^{2}} w_{j}^{2}+w_{0}},
$$

as the left hand-side is negative and the right-hand side is positive. Note that the fact that $g\left(\bar{G}^{1}\right)$ is positive is essential for the above identity to hold. By adding the appropriate terms (not involving $A^{1}$ or $\bar{G}^{1}$ ),

$$
V_{t}^{1}\left(x_{1}, x_{2} \mid A^{1}, A^{2}\right)<V_{t}^{1}\left(x_{1}, x_{2} \mid \bar{G}^{1}, A^{2}\right) .
$$

If $0<g\left(A^{1}\right)<g\left(\bar{G}^{1}\right), A^{1}>\bar{G}^{1}$, just note that

$$
\frac{g\left(A^{1}\right)}{\sum_{j \in A^{1}} w_{j}^{1}+\sum_{j \in A^{2}} w_{j}^{2}+w_{0}} \leq \frac{g\left(\bar{G}^{1}\right)}{\sum_{j \in \bar{G}^{1}} w_{j}^{1}+\sum_{j \in A^{2}} w_{j}^{2}+w_{0}} .
$$

Lemma 2 If a firm, say firm 1 , is in Case II, and if $A_{l}^{1}<\bar{G}^{1}$, then node $l$ is not a best response for any strategy of the other firm.

$\underline{\text { Proof }}$

Let $A^{2}$ be a strategy choice of firm 2 .

Let $A_{l}^{1}<\bar{G}^{1}$, so $g\left(A_{l}^{1}\right)<g\left(\bar{G}^{1}\right)<0(<0$ as we are in Case II where all $g(\cdot)$ are negative). As $A_{l}^{1}<\bar{G}^{1}$

$$
\sum_{j \in A_{l}^{1}} w_{j}^{1}+\sum_{j \in A^{2}} w_{j}^{2}+w_{0}<\sum_{j \in \bar{G}^{1}} w_{j}^{1}+\sum_{j \in A^{2}} w_{j}^{2}+w_{0}
$$

and

$$
\begin{aligned}
\frac{g\left(A_{l}^{1}\right)}{\sum_{j \in A_{l}^{1}} w_{j}^{1}+\sum_{j \in A^{2}} w_{j}^{2}+w_{0}} & <\frac{g\left(A_{l}^{1}\right)}{\sum_{j \in \bar{G}^{1}} w_{j}^{1}+\sum_{j \in A^{2}} w_{j}^{2}+w_{0}} \\
& <\frac{g\left(\bar{G}^{1}\right)}{\sum_{j \in \bar{G}^{1}} w_{j}^{1}+\sum_{j \in A^{2}} w_{j}^{2}+w_{0}}
\end{aligned}
$$

or in other words, $A_{l}^{1}$ is not the best response function. 

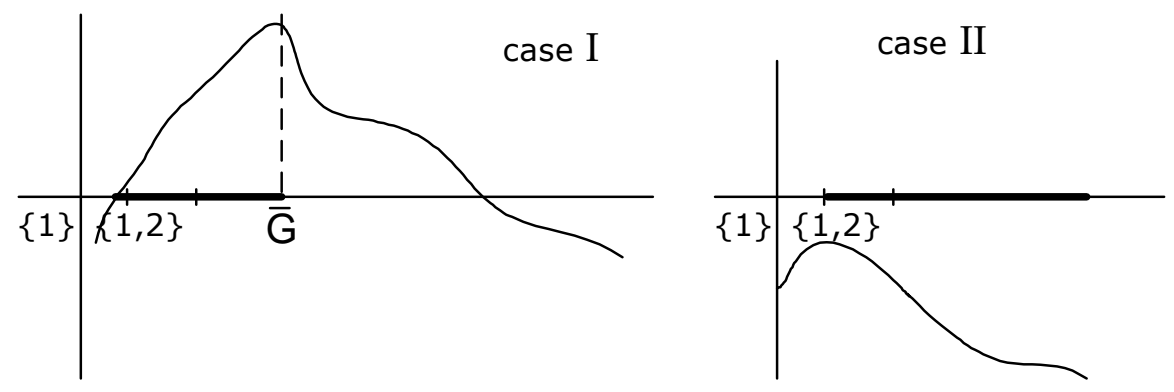

Figure 4: The two cases for the function $g(\cdot)$. Lemmas 1 and 2 show that best-responses lie in the regions marked with bold lines.

Lemma 3 When both firms are in case I, or both are in case II, there exists an ordering of the strategies such that the lowest b-r graph has no crossing arcs.

$\underline{\text { Proof }}$

By Proposition 1 and Lemmas 1 and 2, we can ignore nodes with $g(A)$ negative in Case I and nodes with $A<\bar{G}^{i}$ in Case II.

Both firms are in case I: Order both the strategy spaces by increasing sizes as $A_{1}^{1}, A_{2}^{1}, \ldots, A_{n_{1}}^{1}$ and $A_{1}^{2}, A_{2}^{2}, \ldots, A_{n_{2}}^{2}$ respectively.

By Lemma 1 , both $g\left(A_{k_{1}}\right.$ and $g\left(A_{l_{1}}\right)$ are positive as they have incoming arcs.

So, if firm 2 offers the complete set $k_{2}$, then firm 1's best response is $k_{1}$ and if firm 2 offers the complete set $l_{2}$, then firm 1's best response is $l_{1}$. Because the customer's choice rule is the multinomial logit, this means that:

$$
\frac{g\left(A_{k_{1}}^{1}\right)}{\sum_{j \in A_{k_{1}}} w_{j}^{1}+\sum_{j \in A_{k_{2}}} w_{j}^{2}+w_{0}}>\frac{g\left(A_{l_{1}}^{1}\right)}{\sum_{j \in A_{l_{1}}} w_{j}^{1}+\sum_{j \in A_{k_{2}}} w_{j}^{2}+w_{0}} .
$$

Similarly, when firm 2 offers $l_{2}$,

$$
\frac{g\left(A_{l_{1}}^{1}\right)}{\sum_{j \in A_{k_{1}}} w_{j}^{1}+\sum_{j \in A_{l_{2}}} w_{j}^{2}+w_{0}} \geq \frac{g\left(A_{k_{1}}^{1}\right)}{\sum_{j \in A_{l_{1}}} w_{j}^{1}+\sum_{j \in A_{l_{2}}} w_{j}^{2}+w_{0}} .
$$

Now note that since $k_{1}>l_{1}$, the denominator of the left-hand side of (4) is greater than that of the right-hand side and that this implies $g\left(A_{k_{1}}^{1}\right) \geq g\left(A_{l_{1}}^{1}\right)$, as by Lemma $1, g\left(A_{k_{1}}^{1}\right)>$ 0 . Now if $x / y>z / w$ and $x \geq z, y>0, w>0$, and $x>0$, then $x /(y+v)>z /(w+v)$ for any constant $v>0$.

Consider now,

$$
\frac{g\left(A_{k_{1}}^{1}\right)}{\sum_{j \in A_{k_{1}}^{1}} w_{j}^{1}+\sum_{j \in A_{l_{2}}^{2}} w_{j}^{2}+w_{0}} .
$$


As there are no firm 2's terms in the numerator, and since $l_{2}>k_{2}$, we have the following inequality,

$$
\frac{g\left(A_{k_{1}}^{1}\right)}{\sum_{j \in A_{k_{1}}^{1}} w_{j}^{1}+\sum_{j \in A_{l_{2}}^{2}} w_{j}^{2}+w_{0}}>\frac{g\left(A_{l_{1}}^{1}\right)}{\sum_{j \in A_{l_{1}}^{1}} w_{j}^{1}+\sum_{j \in A_{l_{2}}^{2}} w_{j}^{2}+w_{0}} .
$$

By adding appropriate terms on both sides (not involving the strategy choices of either firm), we have proved:

$$
V_{t}^{1}\left(x_{1}, x_{2} \mid A_{k_{1}}^{1}, A_{l_{2}}^{2}\right)>V_{t}^{1}\left(x_{1}, x_{2} \mid A_{l_{1}}^{1}, A_{l_{2}}^{2}\right) .
$$

But this contradicts the fact that if firm 2 offers $l_{2}$, the best-response set for firm 1 is $l_{1}$-it is in fact $k_{1}>l_{1}$.

Both firms are in Case II: Order the strategy space of firm 1 by decreasing sizes as $A_{n_{1}}^{1}, \ldots, \bar{G}^{1}$ and for firm 2 by increasing sizes as $\bar{G}^{2}, \ldots, A_{n_{2}}^{2}$ respectively.

Suppose there exist two crossing arcs in this ordering. So let $l_{1}<k_{1}$ and $l_{2}>k_{2}$, and the two lowest crossing b-r arcs for firm 1 be $\left(k_{2}, k_{1}\right)$ and $\left(l_{2}, l_{1}\right)$.

As firm 1 has the decreasing order, $g$ is monotonically increasing, from $A_{n_{1}}^{1}$ till $\bar{G}^{1}$, $g\left(A_{l_{1}}^{1}\right) \leq g\left(A_{k_{1}}^{1}\right)<0$.

As $A_{l_{1}}^{1}$ is the best response to $A_{l_{2}}^{2}$,

$$
\frac{g\left(A_{l_{1}}^{1}\right)}{\sum_{j \in A_{l_{1}}^{1}} w_{j}^{1}+\sum_{j \in A_{l_{2}}^{2}} w_{j}^{2}+w_{0}} \geq \frac{g\left(A_{k_{1}}^{1}\right)}{\sum_{j \in A_{k_{1}}^{1}} w_{j}^{1}+\sum_{j \in A_{l_{2}}^{2}} w_{j}^{2}+w_{0}} .
$$

Now if $x / y>z / w$ and $x \leq z<0, y>0, w>0$, then $x /(y-v)>z /(w-v)$ for any constant $v>0$. As firm 2's nodes are ordered by increasing nested sets,

$$
\sum_{j \in A_{l_{2}}^{2}} w_{j}^{2}>\sum_{j \in A_{k_{2}}^{2}} w_{j}^{2}
$$

which implies

$$
\frac{g\left(A_{l_{1}}^{1}\right)}{\sum_{j \in A_{l_{1}}^{1}} w_{j}^{1}+\sum_{j \in A_{k_{2}}^{2}} w_{j}^{2}+w_{0}} \geq \frac{g\left(A_{k_{1}}^{1}\right)}{\sum_{j \in A_{k_{1}}^{1}} w_{j}^{1}+\sum_{j \in A_{k_{2}}^{2}} w_{j}^{2}+w_{0}} .
$$

But this contradicts the fact that $A_{k_{1}}^{1}$ is the best response to $A_{k_{2}}^{2}$.

As similar argument holds for crossing arcs as a response to strategies of firm 1.

So, if both firms are in Case I or both are in Case II, by Theorem 1, there is an equilibrium for the stage. The difficulty is if one firm is in Case I and the other in Case II. Consider the following example. 


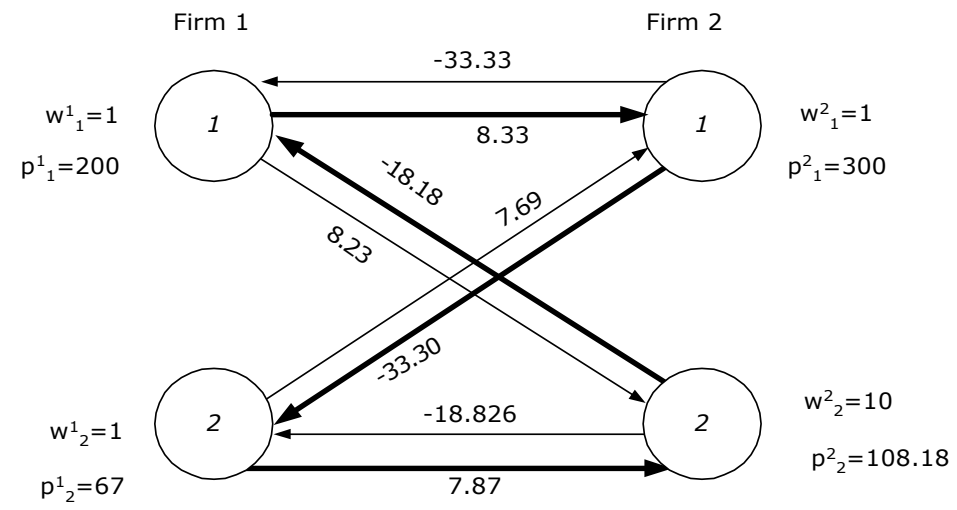

Figure 5: Payoff values for Example 1. Best-responses are in thick lines.

Example: Let firm 1 and firm 2 have the following data.

\begin{tabular}{lll}
\hline & Firm 1 & Firm 2 \\
& $i=1$ & $i=2$ \\
\hline$\Delta$ & 100 & 100 \\
$\delta$ & 50 & 10 \\
$w_{1}^{i}$ & 1 & 1 \\
$w_{2}^{i}$ & 1 & 10 \\
$w_{0}$ & 10 & 10 \\
$p_{1}^{i}$ & 200 & 300 \\
$p_{2}^{i}$ & 67 & 108.18 \\
\hline
\end{tabular}

Then the payoff and best-response arcs are as given in Figure 5, and it can be seen that there is a cycle and no equilibrium, even for the multinomial logit choice function.

So even though the single-period problem always has an equilibrium (Anderson et al. [1]), the competitive game over a finite number of periods, may end up having no equilibrium. Notice that this is not a repeated game - the dynamic program has strong intertemporal relationships, and the parameters of the game changes over time.

We next give some conditions on the choice model parameters that guarantees existence of an equilibrium. Define for $A_{k}>A_{l}$,

$$
q_{A_{k}-A_{l}}^{i}=\frac{\sum_{j \in A_{k}^{i}-A_{l}^{i}} w_{j}^{i} p_{j}^{i}}{\sum_{j \in A_{k}^{i}-A_{l}^{i}} w_{j}^{i}},
$$

which represents the weighted average price for the products from $k$ to $l$. 
Proposition 2 If suppose the parameters of the choice model satisfy, for $i=1,2$, for any $A_{k}^{i}, A_{l}^{i}$ :

$$
\sum_{j \in A_{l}^{i}} p_{j}^{i} w_{j}^{i}>q_{A_{k}-A_{l}}^{i}\left(w_{0}+\sum_{j \in A_{l}^{i}} w_{j}^{i}\right) .
$$

Then there exists a pure-strategy Nash equilibrium in the subsets that each firm offers at every time interval $t$.

$\underline{\text { Proof }}$

From Lemma 3 it only remains to show the non-crossing property when one firm is in Case II and the other firm is in Case I.

We will show that if a firm is in Case II, then if the weights and prices satisfy (9), the only best response for the firm, for any strategy of the other firm, is to offer set $\bar{G}^{i}$.

Say firm 1 is in Case II. Let $A_{k}^{1}$ be a best-response to firm 2's strategy $A_{k}^{2}$, and $A_{k}^{1}>\bar{G}^{1}$ (by Lemma $2, A_{k}^{1} \geq \bar{G}^{1}$ ). So,

$$
0>\frac{g\left(A_{k}^{1}\right)}{\sum_{j \in A_{k_{1}}^{1}} w_{j}^{1}+\sum_{j \in A_{k_{2}}^{2}} w_{j}^{2}+w_{0}}>\frac{g\left(\bar{G}^{1}\right)}{\sum_{j \in \bar{G}^{1}} w_{j}^{1}+\sum_{j \in A_{k_{2}}^{2}} w_{j}^{2}+w_{0}} .
$$

Therefore,

$$
\frac{\sum_{j \in A_{k_{1}}^{1}} w_{j}^{1}+\sum_{j \in A_{k_{2}}^{2}} w_{j}^{2}+w_{0}}{\sum_{j \in \bar{G}^{1}} w_{j}^{1}+\sum_{j \in A_{k_{2}}^{2}} w_{j}^{2}+w_{0}}<\frac{g\left(A_{k}^{1}\right)}{g\left(\bar{G}^{1}\right)}
$$

which implies,

$$
\frac{\sum_{j \in A_{k_{1}}^{1}-\bar{G}^{1}} w_{j}^{1}}{\sum_{j \in \bar{G}^{1}} w_{j}^{1}+\sum_{j \in A_{k_{2}}^{2}} w_{j}^{2}+w_{0}}<\frac{\sum_{j \in A_{k_{1}}-\bar{G}^{1}} w_{j}^{1}\left[p_{j}^{1}-\Delta\right]}{g\left(\bar{G}^{1}\right)}
$$

or,

$$
\sum_{j \in \bar{G}^{1}} w_{j}^{1}+\sum_{j \in A_{k_{2}}^{2}} w_{j}^{2}+w_{0}<\frac{\sum_{j \in \bar{G}^{1}} w_{j}^{1}\left[p_{j}^{1}-\Delta\right]-w_{0} \delta}{q_{A_{k}-\bar{G}^{1}}^{1}-\Delta}
$$

or,

$$
\begin{aligned}
\sum_{j \in A_{k_{2}}^{2}} w_{j}^{2} & <\frac{\sum_{j \in \bar{G}^{1}} w_{j}^{1}\left[p_{j}^{1}-q_{A_{k}-\bar{G}^{1}}^{1}\right]-w_{0}\left[\delta+q_{A_{k}-\bar{G}^{1}}^{1}-\Delta\right]}{q_{A_{k}-\bar{G}^{1}}^{1}-\Delta} \\
& =\frac{\sum_{j \in \bar{G}^{1}} w_{j}^{1} p_{j}^{1}-q_{A_{k}-\bar{G}^{1}}^{1}\left[\sum_{j \in \bar{G}^{1}} w_{j}^{1}+w_{0}\right]-w_{0}[\delta-\Delta]}{q_{A_{k}-\bar{G}^{1}}^{1}-\Delta}
\end{aligned}
$$

Note that $\bar{G}^{i}$, represent the set $A_{l}^{i}$ such that $h\left(A_{l}^{i}\right)=\sum_{j \in A_{l}^{i}} w_{j}^{i}\left[p_{j}^{i}-\Delta\right] \geq 0$, but $h\left(A_{l+1}^{i}\right)<0$. Note that there is at least one $A_{l}^{i}$ with $h\left(A_{l}^{i}\right) \geq 0$ as there is at least 

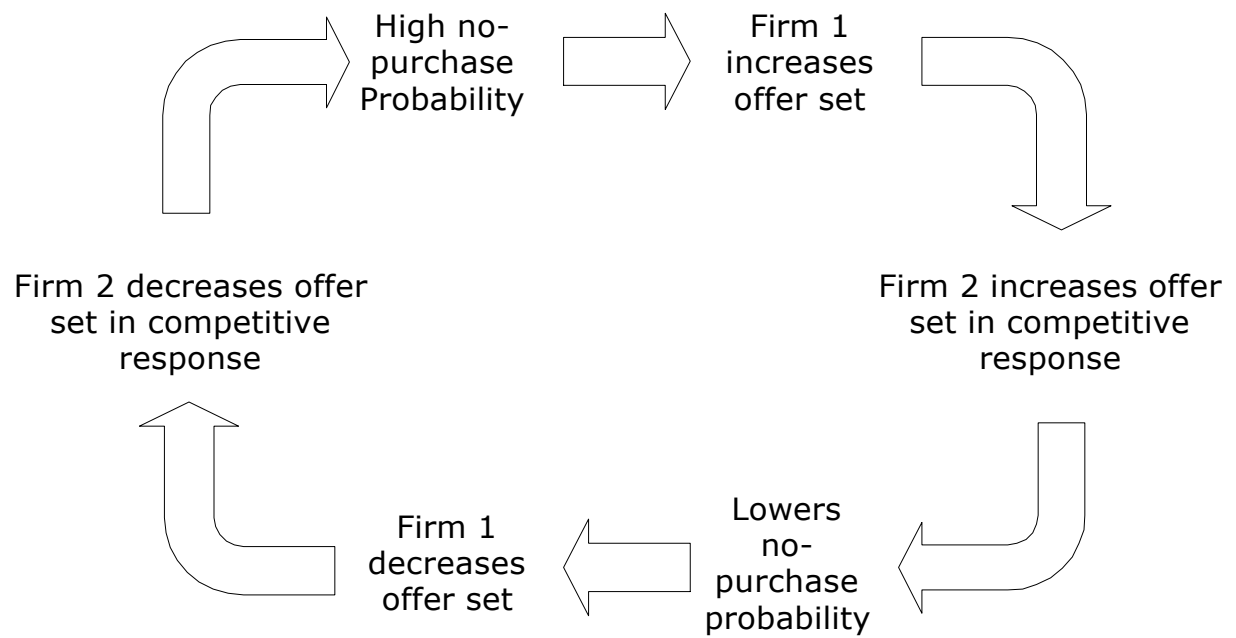

Figure 6: No-purchase probabilities causing a best-response cycle.

one $p_{j}^{i} \geq \Delta$. If $h\left(A_{l}^{i}\right) \geq 0$ for all offer sets of firm i, let $\bar{G}^{i}$ be just the largest offer set, $\left\{1,2, \ldots, n_{i}\right\}$. So the denominator of (10) is less than zero.

From (9) and the fact that $\Delta>\delta$, the numerator is greater than 0 , so the right hand side is less than zero, while the left hand side is greater than 0 . So, $A_{k}^{1}$ cannot be a best-response arc.

So, order the nodes of both firm 1 and 2 by increasing order. (The b-r arcs do not cross (that argument that firm 2's b-r arcs do not cross are the same as in Lemma 3, for the case where both firms are in Case I). So by Theorem 1, there exists an equilibrium.

The condition for Proposition 2 holds for instance, when $w_{0}=0$. That is, if a customer arrives in a period, he is assumed to make a purchase if one of the firms is offering inventory.

In the model of Dudey [4] both firms can observe each other's capacities, and the customer in each period will always purchase from one of the two firms (equivalent to $w_{0}=0$ ). So the no-purchase option plays a crucial role indeed - its relative magnitude determines whether we have an equilibrium in the game or not. Figure 6 shows the intuition behind why modeling the no-purchase option introduces the instability into the game under certain circumstances.

While Example 1 is somewhat discouraging, it is not indicative of a non-equilibrium in many interesting cases, such as the following: if say the two firms have equal capacities, identical products, and customers, then will we see equilibrium in the offer sets? Example 1 is not applicable and it is possible that one can derive conditions on $\Delta$ and $\delta$ to show the existence of an equilibrium. Indeed Example 1 does not even conclusive for the general case - it does not show that the $\Delta$ and $\delta$ used actually arise in a dynamic game. 


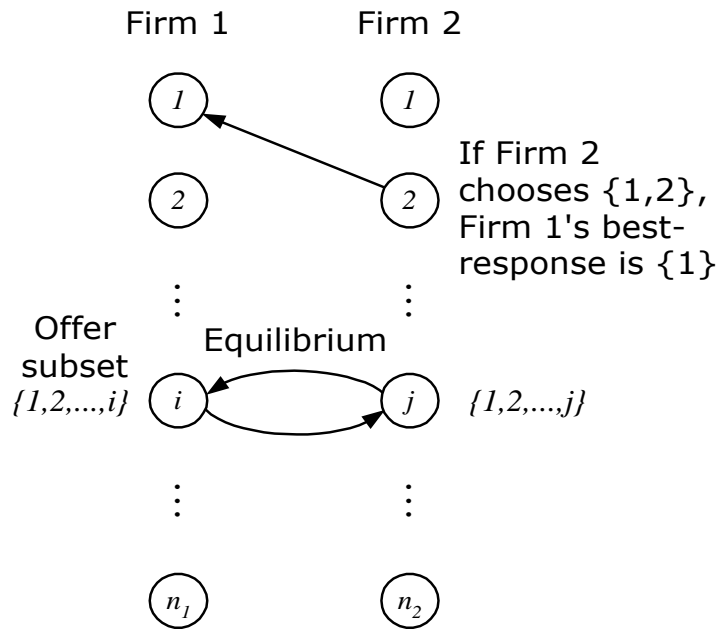

Figure 7: The discrete-choice equilibrium bipartite graph.

\subsection{Unobservable capacities}

This case holds in the airline case, where a firm can observe the competitor's available subset but cannot observe its remaining capacity.

We have briefly discussed the symmetric case earlier, where the firms have identical capacities, products and consumers are indifferent to the two firms. In the presence of a no-purchase alternative, it is not even clear that this game has an equilibrium. However, on average, we would expect both firms to have identical capacities at any given point of time. So Proposition 3 applies and we would expect to see an equilibrium. Indeed, if capacities are not observable, firms may just assume that the rival's capacity is the same as theirs and solve their dynamic programs - this would lead to an equilibrium strategy for both players, as both would be in Case I together or in Case II together.

We consider another case where both firm's essentially ignore the competitor's capacity. The value functions for firm 1 at time $t$ given firm 2 offers $A^{2}$ can then be given by the following:

$$
\begin{aligned}
V_{t}^{1}\left(x \mid A^{2}\right) & = \\
\max _{A^{1} \subseteq 2^{\left|n_{1}\right|}} & \left\{\lambda \sum_{j \in A^{1}} \gamma_{j}^{i}\left(A^{1}, A^{2}\right)\left(p_{j}^{i}-\left(V_{t+1}^{1}(x)-V_{t+1}^{1}(x-1)\right)\right)+V_{t+1}^{1}(x)\right\} .
\end{aligned}
$$

Similarly for firm 2 .

Firms react to the offered subsets, and do not consider competitor's capacity. This is a considerable simplification. It is possible that they may be making some forecasts on competitor remaining capacity, learn from competitor's actions in a Bayesian/learning 
framework etc. Such complicated modeling is beyond the scope of this paper, as here, we are just trying to illustrate an application of the no-crossing technique. ${ }^{1}$

Proposition 3 Let $l_{1}<k_{1}$ and $l_{2}>k_{2}$. Then the lowest b-r graph cannot have the two b-r arcs for firm $1\left(k_{2}, k_{1}\right)$ and $\left(l_{2}, l_{1}\right)$. Similarly for firm 2.

$\underline{\text { Proof }}$

Suppose there exist two such arcs. So, if firm 2 offers the complete set $k_{2}$, then firm 1's best response is $k_{1}$ and if firm 2 offers the complete set $l_{2}$, then firm 1 's best response is $l_{1}$. Because the customer's choice rule is the multinomial logit, this means that:

$$
\frac{\sum_{j \leq k_{1}} w_{j}^{1} p_{j}^{1}}{\sum_{j \leq k_{1}} w_{j}^{1}+\sum_{j \leq k_{2}} w_{j}^{2}+w_{0}}>\frac{\sum_{j \leq l_{1}} w_{j}^{1} p_{j}^{1}}{\sum_{j \leq l_{1}} w_{j}^{1}+\sum_{j \leq k_{2}} w_{j}^{2}+w_{0}} .
$$

Similarly, when firm 2 offers $l_{2}$,

$$
\frac{\sum_{j \leq l_{1}} w_{j}^{1} p_{j}^{1}}{\sum_{j \leq l_{1}} w_{j}^{1}+\sum_{j \leq l_{2}} w_{j}^{2}+w_{0}} \geq \frac{\sum_{j \leq k_{1}} w_{j}^{1} p_{j}^{1}}{\sum_{j \leq k_{1}} w_{j}^{1}+\sum_{j \leq l_{2}} w_{j}^{2}+w_{0}} .
$$

Now note that since $k_{1}>l_{1}$, the denominator of the left-hand side of (12) is greater than that of the right-hand side and that this implies $\sum_{j \leq k_{1}} w_{j}^{1} p_{j}^{1} \geq \sum_{j \leq l_{1}} w_{j}^{1} p_{j}^{1}$. As earlier if $x / y>z / w$ and $x \geq z$, then $x /(y+v)>z /(w+v)$ for any constant $v>0$. Since $l_{2}>k_{2}$,

$$
\frac{\sum_{j \leq k_{1}} w_{j}^{1} p_{j}^{1}}{\sum_{j \leq k_{1}} w_{j}^{1}+\sum_{j \leq l_{2}} w_{j}^{2}+w_{0}}>\frac{\sum_{j \leq l_{1}} w_{j}^{1} p_{j}^{1}}{\sum_{j \leq l_{1}} w_{j}^{1}+\sum_{j \leq l_{2}} w_{j}^{2}+w_{0}} .
$$

But this contradicts the fact that if firm 2 offers $l_{2}$, the best-response set for firm 1 is $l_{1}$-it is in fact $k_{1}>l_{1}$.

So, again, by Theorem 1, we have proved the following:

Theorem 2 There exists a Nash equilibrium in the subsets that each firm offers at every time interval $t$.

We do not exclude multiple equilibria. Assume that firms always choose the highest labeled equilibria (open the most number of classes). This would be a reasonable assumption if both firms start off with their largest sets and converge to an equilibrium based on observations of the other's moves.

Then one can define the concept of equilibrium marginal value as $\pi_{i}=V_{t+1}^{i}(x-1)-$ $V_{t+1}^{i}(x)$ where both firms offer their equilibrium sets during periods $t+1, \ldots, T$. Let $A_{\pi_{i}}$ be the complete sets defined by these equilibrium marginal values (that is composed of all the products with prices higher than $\pi_{i}$ ). Then during period $t$, there exist equilibrium subsets of $A_{\pi_{i}}$, so the $T$-period revenue management game has a (subgame perfect) equilibrium.

\footnotetext{
${ }^{1}$ It may come as a surprise that many airlines currently optimize nothing more complicated than Equation 11; or worse - many just ignore the competition.
} 


\subsection{Dynamic Pricing and Lowest-Open-Fare Choice Model}

The results of this Section also extend to a choice model where customers choose the lowest priced available product in the offer sets. If there is a single segment (as we assumed here) of customers, then this is equivalent to firms offering a single price in each time period, i.e., dynamic pricing. Customers then have three options: no-purchase, purchase at the (lowest) price offered by firm 1 or firm 2 .

We need to impose a few conditions on the parameters of the choice model for our earlier results to go through: the weights are decreasing functions of the price, and $w_{j} p_{j}$ is unimodal. For instance, the logit model with weights of the form $e^{-p / \mu}$ used in [1] (pg., 222) satisfies this property. $\mu>0$ is a diversity factor that if close to zero, models customers indifferent to the product characteristics and who buy based purely on price, whereas when $\mu$ is very high, they are quite indifferent to price and buy each product with equal probability.

Acknowledgment: Thanks to Sjaak Hurkens for pointing out some errors in an earlier draft of this paper, and Garrett van Ryzin for discussions on the application.

\section{References}

[1] S. P. Anderson, A. de Palma, and J-F. Thisse. Discrete Choice Theory of Product Differentiation. MIT Press, Cambridge, MA, 1992.

[2] M. Ben-Akiva and S. Lerman. Discrete Choice Analysis: Theory and Application to Travel Demand. MIT Press, Cambridge, Massachusettes, 1985.

[3] G.P Cachon. Stock wars: inventory competition in a two-echelon supply chain with multiple retailers. Operations Research, 49:658-674, 2001.

[4] M. Dudey. Dynamic Edgeworth-Bertrand competition. The Quarterly Journal of Economics, Nov 1992.

[5] S. A. Lippman and K. F. McCardle. The competitive newsboy. Operations Research, 45(1):54-65, 1997.

[6] S. Netessine and R. A. Shumsky. Revenue management games. Technical report, W. E. Simon Graduate School of Business Administration, University of Rochester, Rochester, NY, 2000.

[7] K. T. Talluri and G. J. van Ryzin. Revenue management under a general discrete choice model of consumer behavior. Working paper, Universitat Pompeu Fabra, Barcelona, 2001.

[8] K. T. Talluri and G. J. van Ryzin. The theory and practice of revenue management. Kluwer Academic Publishers, New York, N.Y, 2003. 
[9] D. M. Topkis. Equilibrium points in nonzerosum n-person submodular games. SIAM Journal of Control and Optimization, 17:773-787, 1979.

[10] X. Vives. Oligopoly Pricing: Old Ideas and New Tools. MIT Press, Cambridge, MA, 1998. 